Background: To evaluate the changes in cerebral oxygenation ( $\mathrm{ScO} 2)$ as measured by near-infrared spectroscopy (NIRS) between bolus doses of phenylephrine and mephentermine when used to treat hypotension in patients undergoing supratentorial tumor surgery under general anesthesia.

Materials and Methods: This was a randomized controlled study including 80 ASA- 1 and -2 patients between 18 and 65 years scheduled for supratentorial tumor surgery. Patients were anesthetized with propofol $(1.5-2 \mathrm{mg} / \mathrm{kg}$ ) and fentanyl $(1.5-2 \mu \mathrm{g} / \mathrm{kg})$; and were randomized after hypotension during anesthesia induction to receive bolus dose of either phenylephrine (100 $\mu \mathrm{g}$ ) or mephentermine (6 mg). Ipsilateral and contralateral ScO2 using NIRS, cardiac output using NICOM, mean arterial pressure, systemic vascular resistance, and heart rate were recorded at hypotension, at drug administration and every minute for a total duration of 10 minutes after achieving desired MAP level with study drug.

Results: Both ipsilateral and contralateral $\mathrm{ScO} 2$ decreased significantly in phenylephrine group after drug administration at all 10-minute time points as compared with hypotension time point $(<0.001)$. While in mephentermine group, ipsilateral and contralateral $\mathrm{ScO} 2$ increased significantly at all time points $(<0.001)$. On intergroup comparison, difference of cerebral oxygenation value between two groups was significant at all time points after hypotension for both ipsilateral and contralateral ScO2 $(p<0.001)$. CO decreased significantly in phenylephrine group $(<0.001)$, while increased significantly in mephentermine group at time points of 3 to 10 minutes $(<0.001)$. Cardiac output had statistically significant effect on $\mathrm{ScO} 2(p=0.005)$.

Conclusions: Associated with changes in cardiac output, $\mathrm{ScO} 2$ decreased after phenylephrine treatment while increased after mephentermine treatment.

\section{A012 Comparison of Effect of Ketofol (Ketamine and Propofol) versus Propofol on Jugular Venous Oxygen Saturation in Moderate to Severe Traumatic Brain Injury: A Prospective Randomized Trial}

Tania Singh, ${ }^{1}$ Nidhi Panda, ${ }^{1}$ Ankur Luthra, ${ }^{1}$ Rajeev Chauhan ${ }^{1}$ ${ }^{1}$ Department of Anaesthesia and Intensive care, Postgraduate Institute of Medical Education and Research (PGIMER), Chandigarh, India

Background: Comparison of effect of ketofol with respect to propofol on jugular venous oxygen saturation when used for maintenance of general anesthesia in moderate to severe traumatic brain injury patients as the primary outcome.

Materials and Methods: A prospective randomized controlled trial was done on 40 TBI patients undergoing decompressive hemicraniectomy. Patients in group $\mathrm{P}$ $(n=20)$ received propofol, while patients in group KP $(n=20)$ received combination of ketamine and propofol (1:5) for maintenance of anesthesia. SjvO2 monitoring, intraoperative hemodynamic parameters, ONSD, brain relaxation score,
S100B levels, MRS at discharge, and extended Glasgow outcome scale (GOSE) at 1 and 3 months were observed.

Results: No statistically significant difference was found with respect to jugular venous oxygen saturation in either group. However, intraoperative hemodynamic parameters were better maintained in patients receiving ketofol compared with those receiving propofol $(p<0.05)$ with decreased requirement of phenylephrine or mephentermine in ketofol group. There was no statistically significant difference in quality of brain relaxation, preoperative and postoperative ONSD, MRS, GOSE at 1 month and 3 months, SB100 levels, and number of ventilator days in the either group.

Conclusions: Ketofol (combination of ketamine and propofol in the ratio 1:5) and propofol similarly maintain cerebral oxygenation. However, hemodynamics appears to be better maintained with ketofol as the maintenance agent.

\section{A013 Effect of Different Surgical Positions on the Changes in Cerebral Venous Drainage (CVD) and Intracranial Pressure (ICP) in Patients Undergoing Elective Neurosurgery \\ Keta Thakkar. ${ }^{1}$ Manikandan S., ${ }^{1}$ Ranganatha, ${ }^{1}$ Praveen C.S. ${ }^{1}$ \\ ${ }^{1}$ Division of Neuroanesthesia and Neurocritical Care, Sree \\ Chitra Tirunal Institute for Medical Sciences and Technology \\ (SCTIMST), Trivandrum, Kerala, India}

Background: Various positions employed to facilitate neurosurgical procedures can compromise the cerebral venous return and can cause increase in ICP. There are no direct indices of CVD. We aimed to use ultrasound (USG) and Doppler indices to assess the changes in CVD during positioning (supine, prone, and lateral) of patients undergoing neurosurgical procedures.

Materials and Methods: After IEC approval, consented ASA I/II patients with GCS 14 to 15 undergoing elective primary brain tumor resection surgery were included. Internal jugular vein (IJV) cross-sectional areas and peak Doppler velocities were recorded on both sides with USG at three different time intervals (before induction of anesthesia (T0), 10 minutes after induction (T1), and 10 minutes after final positioning (T2)). In addition, optic nerve sheath diameter (ONSD) was measured as an estimate of ICP at T1 and T2. Forty-five patients were included in all three groups, that is, supine, lateral, and prone (15 each).

Results: We found a significant change in flow from supine 0 to 30 degrees head tilt. In prone position, in spite of increase in IJV cross-sectional area, flow did not change. In lateral, the dependent side showed a severe decrease in IJV flow $(p<0.05)$. The maximum increase in ONSD was found in lateral position. The right IJV was dominant in $88 \%$ patients.

Conclusion Decrease in IJV flow was seen after final positioning, more significantly in dependent side of lateral position and supine with head tilt. This warrants careful positioning during neurosurgical procedures to prevent iatrogenic increase in ICP which can lead to brain bulge and increased bleeding. 